\title{
Molecular dynamics modeling of cooling of vibrationally highly excited carbon dioxide produced in the photodissociation of organic peroxides in solution $\dagger$
}

\author{
Alexander Kandratsenka, ${ }^{a}{ }^{a}$ Jörg Schroeder, ${ }^{a}$ Dirk Schwarzer ${ }^{a}$ and \\ Vyacheslav S. Vikhrenko ${ }^{b}$ \\ ${ }^{a}$ Abteilung Spektroskopie und Photochemische Kinetik, Max-Planck-Institut für \\ Biophysikalische Chemie, Am Faßberg 11, D-37077 Göttingen, Germany. \\ E-mail: akandra@gwdg.de \\ ${ }^{\boldsymbol{b}}$ Department of Theoretical Mechanics, Belarusian State Technological University, \\ Sverdlova 13a, 220050 Minsk, Belarus
}

Received 21st September 2004, Accepted 14th January 2005

First published as an Advance Article on the web 2nd February 2005

\begin{abstract}
Non-equilibrium (NEMD) and equilibrium (EMD) molecular dynamics simulations are performed to investigate the vibrational cooling and asymmetric stretch spectral evolution of highly excited carbon dioxide produced in the photodissociation of organic peroxides in the solvents dichloromethane, carbon tetrachloride and xenon. Due to strong Fermi resonance the symmetric stretching and bending modes of carbon dioxide in $\mathrm{CH}_{2} \mathrm{Cl}_{2}$ and $\mathrm{CCl}_{4}$ jointly relax on a ten and hundred picosecond timescale, respectively, which is in accordance with experiment. However, the high frequency $\mathrm{CO}_{2}$ asymmetric stretch vibration relaxes on a considerably longer time scale because of weak interaction with the other modes. The relaxation rate coefficients of (and works done by) different modes obtained from NEMD and the Landau-Teller rate coefficients calculated through equilibrium force time correlation functions are in reasonable agreement. The analysis of these results leads to the conclusion that, in contrast to xenon where the relaxation takes about $20 \mathrm{~ns}$, the shorter time scales in $\mathrm{CH}_{2} \mathrm{Cl}_{2}$ and $\mathrm{CCl}_{4}$ are caused by efficient near resonant vibration to vibration energy transfer from carbon dioxide to solvent molecules. The results of the non-equilibrium simulations are used to monitor the quasi-stationary asymmetric stretch infrared spectra of carbon dioxide during the cooling process. Comparison of the corresponding experimental results suggests that carbon dioxide initially is produced with a broad distribution of energy disposed in its bend and symmetric stretch modes while the asymmetric stretch mode remains unexcited.
\end{abstract}

\section{Introduction}

The rapid fragmentation of vibrationally highly excited intermediate radicals is a key process in ultrafast photodissociation reactions that may have a crucial impact on final product distributions in complex chemical reactions. ${ }^{1,2}$ The efficiency of these reaction channels strongly depends on the competing process of cooling 'hot' intermediates by vibrational energy relaxation (VER) in a solvent environment, as exemplified in a recent systematic study of photo-induced decomposition of organic peroxides. ${ }^{3-7}$ In these investigations, UV photo-excitation of a range of organic peroxide compounds in solution was found to cause $\mathrm{O}-\mathrm{O}-$ bond scission on a femtosecond time-scale generating vibrationally highly excited aroyloxy radicals. Based on normal mode frequencies obtained from quantum chemical calculations, statistical rate theory applied to the decarboxylation reaction of the nascent radicals provided a quantitative description of the experimentally observed fragmentation kinetics. The model required, however, ad hoc assumptions about the VER rate of the hot intermediate radicals in liquid $\mathrm{CH}_{2} \mathrm{Cl}_{2}$ and $\mathrm{CCl}_{4}$. In addition, in a series of UV-pump/IR-probe experiments, ${ }^{8-10}$ it was found that the ultrafast decarboxylation reactions of 'hot' aroyloxy radicals in turn generate vibrationally highly excited $\mathrm{CO}_{2}$, whose subsequent VER causes a temporal evolution of the transient IRabsorption of the $\nu_{3}$-band (asymmetric stretch) of $\mathrm{CO}_{2}$ as

$\dagger$ This article is dedicated to Professor Dr Michael Buback on the occasion of his 60 th birthday. illustrated in Fig. 1a. The initially broad absorption band narrows and shifts to the blue on a time-scale of about 100 ps until it attains its room temperature spectral position and shape.

As a first approximation one may assume that the internal energy of $\mathrm{CO}_{2}$ generated in the decarboxylation process can be characterized by a common vibrational temperature for all modes, and that the spectral evolution is caused by anharmonic coupling between normal modes which changes as their level population decreases due to VER in the solvent environment. In such a model, each vibrational temperature gives rise to a specific transient $\nu_{3}$ absorption band of $\mathrm{CO}_{2} \cdot{ }^{10,11} \mathrm{~A}$ comparison of simulated (Fig. 1b) and observed spectra shows rough qualitative agreement concerning the trend of the temporal evolution, but the measured IR-bands tend to be significantly broader.

Evidently, for a better quantitative description of the overall fragmentation reaction a detailed understanding of VER in complex liquids would be highly desirable. This is also of significance in a wider perspective, as $\mathrm{CO}_{2}$ VER is involved in many processes that underly environmental phenomena, industrial and scientific applications in which carbon dioxide plays a mayor role, ${ }^{12-14}$ which is why great efforts have been made to understand its properties and behavior on micro- and macroscopic levels. ${ }^{15,16}$ Extensive investigations of VER of carbon dioxide in the gas phase carried out in the 1950s and 1960 s concerning the importance of this process for $\mathrm{CO}_{2}$-laser operation $^{17-23}$ are summarized in a comprehensive review by Taylor and Bitterman. ${ }^{12}$ The main conclusions from these 

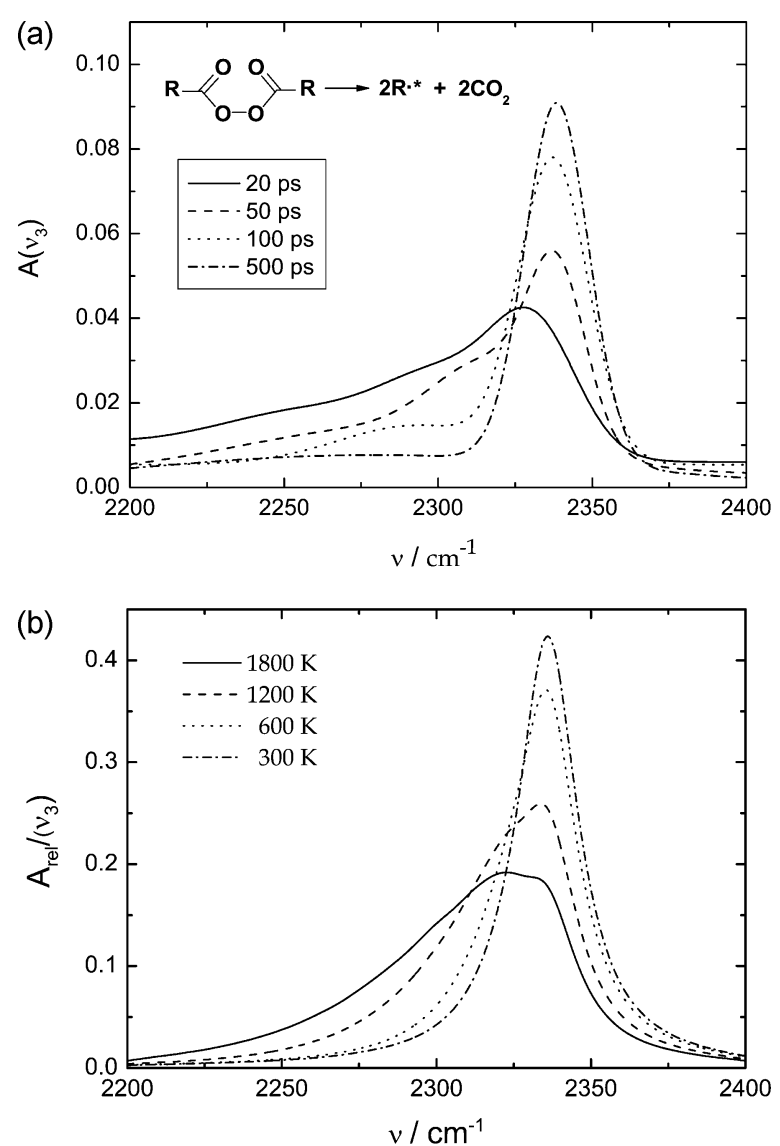

Fig. 1 (a) Picosecond time resolved IR absorption spectra of the carbon dioxide product asymmetric stretch band following UV-photoinduced fragmentation of an organic peroxide (see insert, $\mathrm{R}=4$ methoxyphenyl) at $248 \mathrm{~nm}$ in dichloromethane solution at room temperature (unpublished results from Diploma Thesis of M. Seidel, University of Göttingen, 2000). (b) Simulated carbon dioxide asymmetric stretch IR absorption band in solution at different vibrational temperatures according to an anharmonic coupling model described previously. ${ }^{10,11}$ All vibrational modes, except $\nu_{3}$, are assumed to share a common vibrational temperature.

studies are that (i) vibrational relaxation times of $\mathrm{CO}_{2}$ at room temperature range from a few $\mu \mathrm{s}$ atm to several tens of $\mu \mathrm{s}$ atm depending on the collision partner $\left(\mathrm{CO}_{2}, \mathrm{~N}_{2}, \mathrm{O}_{2}, \mathrm{He}\right.$, rare gases), and (ii) that all vibrational modes of $\mathrm{CO}_{2}$ relax simultaneously. Considering VER of individual vibrational modes one has to distinguish between intramolecular vibrational energy redistribution (IVR) referring to vibrational energy loss as a consequence of energy exchange between normal modes of a given molecule and collisional vibrational energy transfer (CET), by which we denote vibrational energy loss due to intermolecular energy transfer to the bath. Using this terminology, observation (ii) means that IVR is fast (enhanced by strong Fermi-resonance ${ }^{24,25}$ between the bend and symmetric stretch vibrations) compared to the overall VET time scale, and the rate limiting process is CET. Later studies ${ }^{26-31}$ corroborated these findings.

Investigations of carbon dioxide VER in condensed fluids, on the other hand, are scarce. It was shown ${ }^{32}$ that vibrational energy relaxation of $\mathrm{CO}_{2}$ in liquid nitrogen at $70 \mathrm{~K}$ is of submicrosecond duration (around $0.23 \mu \mathrm{s}$ ), and that the probability of vibrational energy state change in a collision calculated on the basis of the independent binary collision (IBC) model is comparable for gas and liquid phase. ${ }^{32}$ However, the time scale of vibrational energy relaxation of highly vibrationally excited $\mathrm{CO}_{2}$ produced in the process of peroxide decomposition in polyatomic solvents $\left(\mathrm{CCl}_{4}\right.$ and $\left.\mathrm{CH}_{2} \mathrm{Cl}_{2}\right)$ was found to be three orders of magnitude faster, i.e. it takes place on a sub-nanosecond time-scale. ${ }^{8-10}$ This probably indicates that the VER mechanisms in these cases are fundamentally different as they involve efficient $\mathrm{V}$-V-transfer routes due to the presence of several solvent intramolecular normal mode frequencies close to the bend and symmetric stretch frequencies of $\mathrm{CO}_{2}$. The details of the VER mechanism of carbon dioxide in liquid solution, however, are not understood, and it seems highly desirable to perform molecular dynamics simulations to improve this situation.

Non-equilibrium molecular dynamics (NEMD) simulations $^{33-38}$ and the Landau-Teller (LT) approach combined with equilibrium molecular dynamics (EMD) simulations ${ }^{39-45}$ are the most widely used theoretical methods for investigating VER of polyatomic molecules in solution. The former is the most straightforward and reliable method when classical mechanics is applicable. The latter is computationally more efficient and can be used to incorporate quantum mechanical corrections, although, the exact form of the quantum correction factor is not known. ${ }^{46,47}$

To study VER of highly excited carbon dioxide in different liquid solvents without $(\mathrm{Xe})$ and with $\left(\mathrm{CCl}_{4}\right.$ and $\left.\mathrm{CH}_{2} \mathrm{Cl}_{2}\right)$ internal structure, we apply both classical equilibrium and non-equilibrium molecular dynamics simulations. Classical simulation results can be quite appropriate in situations when near resonant $\mathrm{V}-\mathrm{V}$ energy transfer dominates the relaxation because energy transfer can be considered as vibrational energy relaxation of a harmonic oscillator in a harmonic bath, a model that is known to be described exactly by the corresponding classical system. ${ }^{48}$ We will address several fundamental questions concerning the VER time scales, the pathways of energy flow from vibrationally excited $\mathrm{CO}_{2}$ to different solvents, the significance of vibration-to-vibration contributions to intermolecular energy transfer, and the applicability of LT type expressions to VER rates of polyatomic molecules under different conditions. The results can be used for subsequent quantum mechanical considerations, because of the close correspondence between classical and quantum mechanical treatments of vibrational motion. ${ }^{49,50}$

The paper is organized as follows. In the next section basic theoretical expressions applicable to the description of vibrational energy relaxation and the investigation of vibrational spectra of polyatomic molecules are considered. Section 3 contains information about inter- and intramolecular potentials used and details of the computer simulations. The simulation results, their comparison with experimental data and their discussion are given in Section 4.

\section{Theoretical background}

The vibrational energy of the solute is represented as the sum over all $n_{\mathrm{s}}$ vibrational degrees of freedom of mode energies $E_{\alpha}$, which contain all the terms comprising solely coordinate $\alpha$, and the mode interaction term $E^{\prime}$

$$
E_{\mathrm{vibr}}=\sum_{\alpha=1}^{n_{\mathrm{s}}} E_{\alpha}+E^{\prime} .
$$

The relaxation rates $k$ and $\tilde{k_{\alpha}}$ for the total and mode specific vibrational energies, respectively, can be directly obtained from a NEMD simulation by tracing the time evolution of their nonequilibrium values averaged over a set of trajectories. Of course, due to inter-mode interactions some uncertainty in the assignment of the mode specific energies does exist. However, usually the interaction term makes up only a small part of the total vibrational energy even at quite strong vibrational excitation such that this uncertainty is of minor importance. Note, that in general $\tilde{k_{\alpha}}$ depends on energy loss caused by intermolecular CET as well as IVR.

The analysis of how different modes participate in CET is based on the evolution equation for the total vibrational 
energy $^{37,51}$

$$
\begin{gathered}
\dot{E}_{\text {vibr }}=N=\sum_{\alpha=1}^{n_{\mathrm{s}}} N_{\alpha}, \\
N_{\alpha}=Q_{\alpha} \dot{q}_{\alpha}
\end{gathered}
$$

where $N$ is the capacity of intermolecular vibrational energy transfer, $N_{\alpha}$ is the fractional capacity of mode $\alpha$, while $\dot{q}_{\alpha}$ and $Q_{\alpha}$ are the mass-weighted solute normal coordinate and the external normal force exerted by the solvent, respectively. The total solute vibrational energy loss after time $t$ is equal to the corresponding work:

$$
W_{\text {vibr }}(t)=\sum_{\alpha=1}^{n_{\mathrm{s}}} W_{\alpha}(t),
$$

where $W_{\alpha}$ denotes the work term for mode $\alpha$ :

$$
W_{\alpha}(t)=\int_{0}{ }^{t} Q_{\alpha} \dot{q}_{\alpha} \mathrm{d} t .
$$

From the relaxation rate constant $k=\tau^{-1}$ of the total energy and the relative energy transfer efficiencies of individual modes, defined by the ratio $W_{\alpha} / W_{\text {vibr }}$, specific CET rate constants $k_{\alpha}=$ $\tau_{\alpha}{ }^{-1}$ can be calculated. Provided the modes are strongly coupled and their heat capacities are approximately equal, the overall relaxation rate constant is given by the sum of the individual CET rates divided by the number of modes:

$$
k=\frac{1}{n_{\mathrm{s}}} \sum_{\alpha=1}^{n_{\mathrm{s}}} k_{\alpha} .
$$

Combining eqns. (2.4) and (2.6) the mode specific CET rate constant is given by

$$
k_{\alpha}=n_{\mathrm{s}} k \frac{W_{\alpha}}{W_{\text {vibr }}}
$$

CET rate constants can also be calculated from classical EMD simulations. ${ }^{42-44,52}$ We employ here a conventional Landau-Teller type expression for the diagonal matrix elements

$$
\begin{gathered}
k_{\alpha}=\beta \int_{0}^{\infty} \zeta_{\alpha \alpha}(t) \cos \left(\omega_{\alpha} t\right) \mathrm{d} t, \\
\zeta_{\alpha \alpha}(t)=\left\langle Q_{\alpha}(0) Q_{\alpha}(t)\right\rangle,
\end{gathered}
$$

where $\omega_{\alpha}$ is the vibrational frequency of mode $\alpha$ and $\beta=$ $\left(k_{\mathrm{B}} T\right)^{-1}$ is the inverse thermodynamic temperature. (It is worth noting that the solute atom masses do not enter this equation because the normal coordinates are mass weighted.) The calculation of the force time correlation function (TCF) of eqn. (2.9) involves EMD simulations with the solute internal degrees of freedom frozen at equilibrium geometry.

As mentioned in the Introduction the $\mathrm{CO}_{2}$ infrared absorption spectra were measured for investigating the cooling process. The NEMD approach can be used to simulate the temporal evolution of the infrared absorption cross section $\alpha(\omega)$. According to linear response theory ${ }^{53}$

$$
\alpha(\omega)=\frac{4 \pi^{2} \omega^{2} \beta}{3 \operatorname{cn}(\omega)} I(\omega)
$$

where $c$ is the speed of light, $n(\omega)$ is the refraction index of the medium and

$$
I(\omega)=(2 \pi)^{-1} \int_{-\infty}^{\infty} \mathrm{d} t \mathrm{e}^{-\mathrm{i} \omega t}\langle\boldsymbol{\mu}(0) \cdot \boldsymbol{\mu}(t)\rangle
$$

is the absorption lineshape defined in terms of the TCF of the dipole moment $\boldsymbol{\mu}$ of the system.

Eqn. (2.10) for the classical absorption cross section differs from its quantum counterpart by the factor

$$
D(\omega)=\frac{1-\mathrm{e}^{-\beta \hbar \omega}}{\beta \omega \hbar} C(\omega)
$$

in which $C(\omega)$ is the quantum correction factor for the lineshape $I(\omega)$. In the case of harmonic oscillators ${ }^{48}$

$$
C(\omega)=\frac{\beta \omega \hbar}{1-\mathrm{e}^{-\beta \hbar \omega}}
$$

such that $D(\omega)=1$ and, therefore, quantum and classical vibrational spectra agree. Because the fundamental vibrational frequencies of $\mathrm{CO}_{2}$ are rather high, we expect $D(\omega)$ to be close to unity (which is supported by calculations on water ${ }^{54}$ ), such that we may use the classical expression (2.10) to calculate vibrational spectra.

\section{Computational details}

\subsection{Intramolecular potential energy}

3.1.1. $\mathrm{CO}_{2} \cdot \mathrm{CO}_{2}$ is a linear molecule with four internal degrees of freedom: symmetric and asymmetric stretch, and doubly degenerate bend. Thus, its ground state potential energy can be represented in terms of three intramolecular coordinates. For our calculations we chose Chedin's potential energy surface ${ }^{55,56}$ It is expressed in terms of Chedin's coordinates $R_{1}, R_{2}$ and $R_{3}$ which correspond to the symmetric stretch, bend and asymmetric stretch deformations of the molecule, respectively:

$$
\begin{aligned}
& R_{1}=a_{1}\left(2-r_{\mathrm{eq}} \frac{r_{21}+r_{23}}{r_{21} r_{23}}\right), R_{2}=a_{2} \alpha, \\
& R_{3}=a_{3} r_{\mathrm{eq}} \frac{r_{21}-r_{23}}{r_{21} r_{23}},
\end{aligned}
$$

where $r_{21}$ and $r_{23}$ are the bond lengths between the oxygen (labeled by 1 and 3 ) and carbon (labeled by 2 ) atoms, $\pi-\alpha$ is the angle between these bonds, $r_{\mathrm{eq}}$ is the equilibrium bond length (the equilibrium distance between the carbon and oxygen atoms), $a_{j}$ are constants defined by convergence considerations. ${ }^{56}$ Chedin's coordinates attain finite values at infinite bond lengths.

For the potential energy we considered terms up to 6 th order in Chedin's coordinates. The mode specific potential energies are represented as

$$
\begin{aligned}
& U_{1}=\sum_{i=2}^{6} F_{i 00} R_{1}^{i}, \\
& U_{2}=F_{020} R_{2}^{2}+F_{040} R_{2}^{4}+F_{060} R_{2}^{6}, \\
& U_{3}=F_{002} R_{3}^{2}+F_{004} R_{3}^{4}+F_{006} R_{3}^{6} .
\end{aligned}
$$

The remaining terms represent inter-mode interactions of the potential energy

$$
U^{\prime}{ }_{\text {int }}=\sum_{i+j+k=3}^{6} F_{i j k} R_{1}^{i} R_{2}^{j} R_{3}^{k} .
$$

Because of symmetry conditions $j$ and $k$ are even numbers. In the last sum at least two numbers out of $(i, j, k)$ are not equal to zero.

Force constants $F_{i j k}$ that appear in Chedin's potential are determined from spectroscopic measurements. ${ }^{56}$ Normal mode coordinates are constructed by considering the quadratic terms in eqns. (3.15) and retaining the linear parts in Chedin's coordinates (3.14). All calculations ${ }^{57}$ are performed in the Eckart frame ${ }^{58,59}$ to minimize rotation-vibration coupling.

3.1.2. $\mathbf{C C l}_{4}$ and $\mathbf{C H}_{2} \mathbf{C l}_{2}$. The intramolecular potential energy of a given polyatomic solvent is chosen to correctly represent its spectral properties, i.e. by accounting for the interaction between local modes. To this end the representation of an intramolecular potential in terms of symmetry 
coordinates $S_{\alpha}$ seems to be most suitable ${ }^{59}$

$$
U_{\text {solvent }}=\sum_{\alpha, \beta=1}^{3 N-6} F_{\alpha \beta} S_{\alpha} S_{\beta},
$$

where $N$ is the number of atoms of the solvent molecule. Symmetry coordinates $S_{\alpha}$ are defined in terms of linear combinations of local coordinates $\ell_{\tau}$ (bond distances and valence angles)

$$
S_{\alpha}=\sum_{\tau=1}^{N_{\text {local }}} A_{\alpha \tau} \ell_{\tau}
$$

Harmonic force fields of this type are used in this article for tetrachloromethane ${ }^{60}$ and dichloromethane ${ }^{61}$ solvent molecules.

\subsection{Intermolecular potential energy}

The interaction energies between atoms of different molecules are modeled by the sum of conventional Lennard-Jones and Coulomb potentials

$$
U\left(r_{i j}\right)=4 \varepsilon_{i j}\left[\left(\frac{\sigma_{i j}}{r_{i j}}\right)^{12}-\left(\frac{\sigma_{i j}}{r_{i j}}\right)^{6}\right]+\frac{q_{i} q_{j}}{r_{i j}},
$$

where $r_{i j}$ is the distance between atoms $i$ and $j$ carrying the charges $q_{i}$ and $q_{j}$, respectively; $\varepsilon_{i j}$ and $\sigma_{i j}$ are Lennard-Jones parameters. Their values for $\mathrm{Xe},{ }^{62} \mathrm{CH}_{2} \mathrm{Cl}_{2}{ }^{63}$ and $\mathrm{CCl}_{4},{ }^{64,65}$ optimized to correctly model the thermodynamical properties of the neat solvents are collected in Table $1 . \mathrm{CO}_{2}$ interaction parameters are taken from a standard database ${ }^{66}$ without any adjustment. As their values significantly influence the simulation of the relaxation process, ${ }^{37}$ it is desirable to keep a consistent set for all solvents. Parameters for interaction between unlike atoms are calculated using Lorentz-Berthelot mixing rules. ${ }^{67}$

\subsection{Molecular dynamics simulation details}

The simulations were performed with a modified version of the program Moldy ${ }^{68}$ to include the internal motion of molecules.

\begin{tabular}{|c|c|c|c|}
\hline Atom & $\varepsilon_{i j} / \mathrm{kJ} \mathrm{mol}^{-1}$ & $\sigma_{i j} / \AA$ & $q_{i} / \mathrm{e}$ \\
\hline \multirow[t]{2}{*}{$\mathrm{Xe}$} & 2.348 & 3.89 & \\
\hline & $\mathrm{CH}_{2} \mathrm{Cl}_{2}$ & & \\
\hline $\mathrm{C}$ & 0.424 & 3.35 & 0.022 \\
\hline $\mathrm{H}$ & 0.112 & 2.75 & 0.098 \\
\hline \multirow[t]{2}{*}{$\mathrm{Cl}$} & 1.455 & 3.35 & -0.109 \\
\hline & $\mathrm{CCl}_{4}$ & & \\
\hline $\mathrm{C}$ & 0.426 & 4.60 & -0.404 \\
\hline \multirow[t]{2}{*}{$\mathrm{Cl}$} & 0.851 & 3.50 & 0.101 \\
\hline & $\mathrm{CO}_{2}$ & & \\
\hline $\mathrm{C}$ & & & 0.692 \\
\hline \multirow[t]{2}{*}{$\mathrm{O}$} & & & -0.346 \\
\hline & Solute-solvent & & \\
\hline $\mathrm{C}-\mathrm{C}$ & 0.628 & 3.56 & \\
\hline $\mathrm{C}-\mathrm{H}$ & 0.229 & 2.67 & \\
\hline $\mathrm{C}-\mathrm{Cl}$ & 0.852 & 3.60 & \\
\hline $\mathrm{O}-\mathrm{C}$ & 0.725 & 3.21 & \\
\hline $\mathrm{O}-\mathrm{H}$ & 0.265 & 2.32 & \\
\hline $\mathrm{O}-\mathrm{Cl}$ & 0.984 & 2.85 & \\
\hline
\end{tabular}
In this program the predictor-corrector scheme is used to

Table 1 Lennard-Jones and Coulomb potential parameters integrate the equations of motion and the Ewald Sum technique is used to handle long-range Coulomb interactions.

Simulations were performed for one $\mathrm{CO}_{2}$ molecule in (a) 40 $\mathrm{CH}_{2} \mathrm{Cl}_{2}$ molecules at a density of $1.35 \mathrm{~g} \mathrm{~cm}^{-3}$; (b) $40 \mathrm{CCl}_{4}$ molecules at $1.70 \mathrm{~g} \mathrm{~cm}^{-3}$; (c) $200 \mathrm{Xe}$ atoms at $3.0 \mathrm{~g} \mathrm{~cm}^{-3}$.

Before starting the simulation the systems were equilibrated at the desired temperature of $300 \mathrm{~K}$. Then the $\mathrm{CO}_{2}$ molecule at a given configuration was excited with an initial kinetic energy of $87.5 \mathrm{~kJ} \mathrm{~mol}^{-1}$. In the photodissociation experiment ${ }^{8-10}$ the level of excitation of the $\mathrm{CO}_{2}$ molecule and the distribution of excess energy among the modes is unknown. Therefore, in our calculations different cases were investigated. Because of the strong coupling between bend and symmetric stretch modes their relative energy distribution is unimportant. Thus, attention is paid to cases where the asymmetric stretch mode is excited or unexcited. Simulations were performed with the initial kinetic energy distributed equally between the bend, symmetric and asymmetric stretch modes. Additionally, simulations were performed with $85 \mathrm{~kJ} \mathrm{~mol}^{-1}$ in symmetric stretch and bend modes and $2.5 \mathrm{~kJ} \mathrm{~mol}^{-1}$ in the asymmetric stretch mode corresponding to unexcited asymmetric stretch.

The time step of integration was chosen by taking into account the highest vibrational frequency in the simulated system as $0.25 \mathrm{fs}$ in the case of $\mathrm{CH}_{2} \mathrm{Cl}_{2}$ and $0.5 \mathrm{fs}$ for the other solvents. The total simulation time was taken to cover the relaxation process: 250 ps for $\mathrm{CH}_{2} \mathrm{Cl}_{2}, 1 \mathrm{~ns}$ for $\mathrm{CCl}_{4}$ and $10 \mathrm{~ns}$ for xenon. The information necessary to calculate the total and mode-specific solute energy and work as well as the dipole moment was saved every 2 fs (approximately the seventh part of the period of the $\mathrm{CO}_{2}$ fastest mode). The calculated quantities then were averaged over 100 trajectories.

Likewise, for $\mathrm{CH}_{2} \mathrm{Cl}_{2}$ calculations with 80 solvent molecules were performed. The results did not differ from those obtained with the smaller system indicating that 40 solvent molecules are sufficient to properly evaluate the CET dynamics.

To obtain TCFs (2.9) for calculating rates in the framework of the Landau-Teller approach, EMD simulations with rigid $\mathrm{CO}_{2}$ in its equilibrium geometry were performed. Trajectories with a length of $8 \times 10^{6}$ time steps $(2 \mathrm{~ns})$ for the solvent $\mathrm{CH}_{2} \mathrm{Cl}_{2}, 2 \times 10^{7}$ time steps (10 ns) for $\mathrm{CCl}_{4}$ and $8 \times 10^{6}$ time steps ( $4 \mathrm{~ns}$ ) for Xe were generated. To study the influence of electrostatic interactions on the VER rates, calculations in $\mathrm{CH}_{2} \mathrm{Cl}_{2}$ and $\mathrm{CCl}_{4}$ were also conducted with zero partial charges.

\section{Simulation results and discussion}

\subsection{Non-equilibrium MD simulations}

Figs. 2-4 represent NEMD results derived from simulations with charged particles and show time dependencies of the $\mathrm{CO}_{2}$ vibrational total energy and its mode-specific parts in $\mathrm{CH}_{2} \mathrm{Cl}_{2}$ (Figs. 2 and 3) and $\mathrm{CCl}_{4}$ (Fig. 4). The main feature of all simulations is that the energy transfer to the bath is accomplished by the joint relaxation of the strongly coupled bend and symmetric stretch modes. The energy exchange between these modes occurs on a sub-picosecond time scale ${ }^{57}$ such that during relaxation both modes remain in quasi equilibrium. Since the bend vibration is twofold degenerate it contains twice as much energy as the symmetric stretch mode. Small apparent deviations from energy equipartitioning between these modes can be attributed to the interaction term $E^{\prime}$ (eqn. (2.1)) causing some uncertainty in the assignment of the mode specific energy on the normal mode basis.

The asymmetric stretch vibration, on the other hand, is only weakly coupled to the other modes and remains nearly inactive during the relaxation process, i.e. its energy is almost constant. This finding does not depend on whether the asymmetric stretch vibration is initially excited (Fig. 3) or not (Figs. 2 and 4). As a result, the degree of asymmetric stretch excitation 




Fig. 2 Time dependence of the vibrational energy of $\mathrm{CO}_{2}$ and its vibrational modes in $\mathrm{CH}_{2} \mathrm{Cl}_{2}$ with the asymmetric stretch mode initially unexcited: symmetric stretch energy $E_{1}$, bend energy $E_{2}$, asymmetric stretch energy $E_{3}$, total vibrational energy of $\mathrm{CO}_{2} E_{\text {vibr }}$, the sum of bend and symmetric stretch energies $E_{1}+E_{2}$. The dashed line shows the exponential fit to $E_{\mathrm{vibr}}$.

does not influence the relaxation rates of the symmetric stretch/ bend vibrational energy. Consequently, the total energy relaxes on two time scales when all the modes are initially excited (Fig. 3): a faster one determined by the effective energy transfer of the symmetric stretch and bend modes and a slower one due to energy loss of the asymmetric stretch vibration. The latter process is not clearly resolved in Fig. 3 because it takes several ns. We will show below that the energy loss of the $\nu_{3}$ mode mainly reflects IVR to the other modes and does not involve CET to the solvent. A comparison of the two distinct vibrational cooling time scales with the measured values of around 100 ps (see Fig. 1a) suggests that the experimentally observed evolution of the $\mathrm{CO}_{2}$ spectrum after photo-induced fragmentation of organic peroxides arises from the combined relaxation of symmetric stretch and bend vibrations. Therefore, in the following we will concentrate on just this process and neglect the $\nu_{3}$ dynamics.

The total energy decays were fitted by exponentials (dashed lines in Figs. 2-4) with CET times summarized in Table 2. The energy transfer is fastest in $\mathrm{CH}_{2} \mathrm{Cl}_{2}(\tau=62 \mathrm{ps})$, considerably slower in $\mathrm{CCl}_{4}(\tau=344 \mathrm{ps})$, and slowest in xenon $(\tau \cong 20 \mathrm{~ns}$, corresponding energy decay curves are not shown). This behavior nicely correlates with the presence of acceptor vibrational modes of the solvent which are near resonant to the $\mathrm{CO}_{2}$ bend and symmetric stretch vibrations suggesting that the

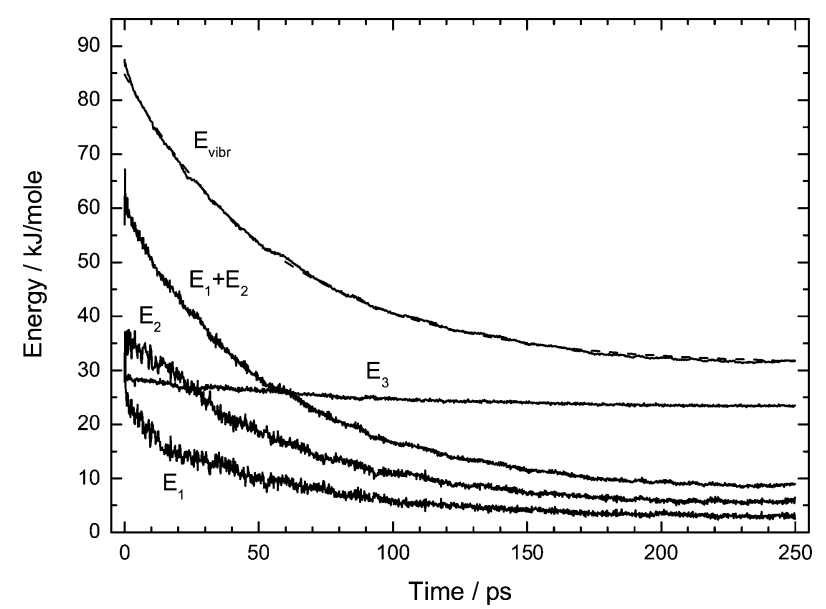

Fig. 3 Time dependence of the vibrational energy of $\mathrm{CO}_{2}$ and its vibrational modes in $\mathrm{CH}_{2} \mathrm{Cl}_{2}$ with the asymmetric stretch mode initially excited. The notations are the same as in Fig. 2.

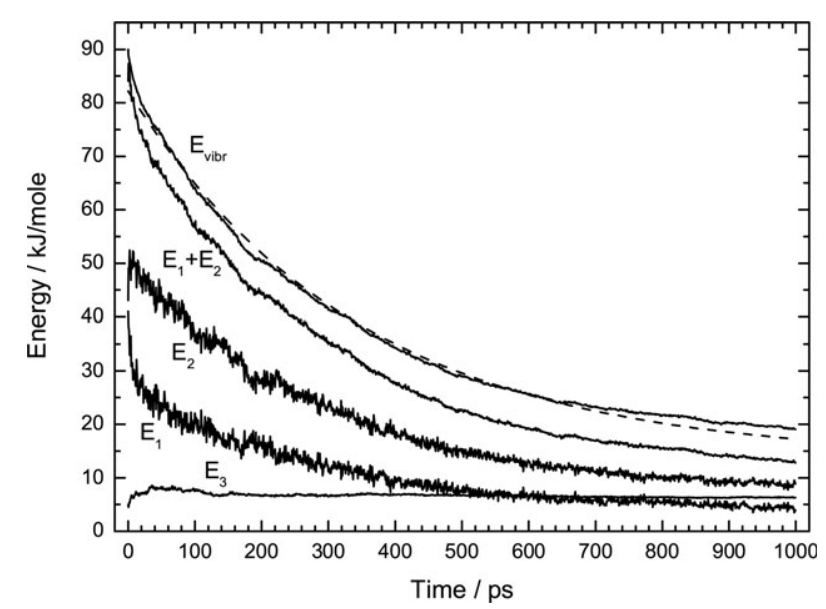

Fig. 4 Time dependence of the vibrational energy of $\mathrm{CO}_{2}$ and its vibrational modes in $\mathrm{CCl}_{4}$ with the asymmetric stretch mode initially unexcited. The notations are the same as in Fig. 2.

relaxation in the polyatomic solvents is dominated by efficient $\mathrm{V}-\mathrm{V}$ energy transfer. $\mathrm{CH}_{2} \mathrm{Cl}_{2}$, e.g., possesses a vibrational frequency which is only about $40 \mathrm{~cm}^{-1}$ off-resonant to the $\mathrm{CO}_{2}$ bend mode (see Table 3). At the same time the $\mathrm{CO}_{2}$ symmetric stretch mode can effectively interact with the 1270 and $1434 \mathrm{~cm}^{-1}$ vibrations of $\mathrm{CH}_{2} \mathrm{Cl}_{2}$, as its spectrum is split due to resonance and nonlinear interaction with the bend mode. Fig. 5 shows that this splitting at high excess energy is around $200 \mathrm{~cm}^{-1}$ while after relaxation it is only $100 \mathrm{~cm}^{-1}$ which is close to the gas phase value at room temperature.

For $\mathrm{CCl}_{4}$, the vibrational mode whose frequency comes closest to that of the $\mathrm{CO}_{2}$ bend still is by almost $100 \mathrm{~cm}^{-1}$ off-resonant. Additionally, $\mathrm{CCl}_{4}$ has no modes which can interact directly with the $\mathrm{CO}_{2}$ symmetric stretch vibration. As a result the relaxation in $\mathrm{CCl}_{4}$ is almost 6 times slower than in $\mathrm{CH}_{2} \mathrm{Cl}_{2}$. In xenon, finally, only $\mathrm{V}-\mathrm{T}$ energy transfer is possible leading to a very long relaxation time constant of about $20 \mathrm{~ns}$. This number involves a considerable error because the trajectories were followed for only $10 \mathrm{~ns}$.

In Table 2 total relaxation times from our NEMD simulations are compared with values derived from recent experiments in $\mathrm{CH}_{2} \mathrm{Cl}_{2}$ and $\mathrm{CCl}_{4}{ }^{8-10}$ For $\mathrm{CH}_{2} \mathrm{Cl}_{2}$ the calculated energy transfer is $13 \%$ too fast whereas for $\mathrm{CCl}_{4}$ it is too slow by more than a factor of 1.5. However, considering the fact that no parameters have been adjusted for these simulations the agreement between theory and experiment is not too bad. In particular, the trend of the relaxation rate when changing the solvent from $\mathrm{CH}_{2} \mathrm{Cl}_{2}$ to $\mathrm{CCl}_{4}$ is correctly reproduced.

According to eqn. (2.5) the activity of a given $\mathrm{CO}_{2}$ vibration in transferring energy to the bath can be monitored by calculating the mode specific work. The time evolution of the corresponding work terms are shown in Figs. 6 and 7 for $\mathrm{CH}_{2} \mathrm{Cl}_{2}$ and $\mathrm{CCl}_{4}$, respectively. In the case of $\mathrm{CH}_{2} \mathrm{Cl}_{2} 74 \%$ of the excess energy is transferred to the solvent via the $\mathrm{CO}_{2}$ bend vibration. The rest is released by the symmetric stretch mode. In contrast, the asymmetric stretch is completely inactive. This is also the case when the asymmetric stretch vibration is initially excited (not shown here) indicating that the slow energy loss of $\nu_{3}$ observed in Fig. 3 is entirely due to IVR.

In $\mathrm{CCl}_{4}$ only the $\mathrm{CO}_{2}$ bend vibration is active in transferring energy to the solvent. This difference to $\mathrm{CH}_{2} \mathrm{Cl}_{2}$ is in complete agreement with the analysis given above where we pointed out that $\mathrm{CCl}_{4}$ does not posses near resonant vibrations to the $\mathrm{CO}_{2}$ symmetric stretch mode allowing for direct $\mathrm{V}-\mathrm{V}$ energy transfer. Also in xenon the energy release takes place only via the $\mathrm{CO}_{2}$ bend channel. This can be expected because in an atomic solvent the energy transfer efficiency decreases nearly exponentially with increasing oscillator frequency (see below). 
Table 2 Mode specific and total CET times obtained from NEMD and LT methods and experiment

\begin{tabular}{|c|c|c|c|c|c|c|c|c|}
\hline \multirow[t]{2}{*}{ Solvent } & \multicolumn{2}{|l|}{$\tau_{1} / \mathrm{ps}$} & \multicolumn{2}{|l|}{$\tau_{2} / \mathrm{ps}$} & \multirow{2}{*}{$\begin{array}{l}\tau_{3} / \mathrm{ps} \\
\mathrm{LT}\end{array}$} & \multicolumn{3}{|l|}{$\tau / \mathrm{ps}$} \\
\hline & NEMD & LT & NEMD & LT & & NEMD & $\mathrm{LT}$ & Exp. $^{a}$ \\
\hline $\mathrm{CH}_{2} \mathrm{Cl}_{2}$ & 76 & 75 & 56 & 50 & $3.1 \times 10^{3}$ & 62 & 56 & 70 \\
\hline $\mathrm{CH}_{2} \mathrm{Cl}_{2}, q_{i}=0$ & 194 & 181 & 53 & 45 & $8.9 \times 10^{3}$ & 71 & 60 & \\
\hline $\mathrm{CCl}_{4}$ & & $9.4 \times 10^{4}$ & 230 & 275 & & 344 & 412 & 200 \\
\hline $\mathrm{CCl}_{4}, q_{i}=0$ & & $1.1 \times 10^{5}$ & 368 & 317 & & 531 & 474 & \\
\hline $\mathrm{Xe}$ & & $6.6 \times 10^{7}$ & & $1.5 \times 10^{4}$ & & $\sim 2.0 \times 10^{4}$ & $2.3 \times 10^{4}$ & \\
\hline
\end{tabular}

${ }^{a}$ Vibrational temperature cooling times from ref. 10 .

Table 3 The solute and solvent fundamental vibrational frequencies (near resonant modes are indicated in bold)



Since symmetric stretch and bend vibrations in $\mathrm{CO}_{2}$ are strongly coupled we can apply eqn. (2.7) to calculate the mode specific CET rate constants of $\nu_{1}$ and $\nu_{2}$ from our NEMD simulations, i.e.

$$
k_{1}=3 k \frac{W_{1}}{W_{\text {vibr }}}, k_{2}=\frac{3 k}{2} \frac{W_{2}}{W_{\text {vibr }}}
$$

where the factor of $1 / 2$ for $k_{2}$ arises from the degeneracy of the bend mode. The corresponding time constants are summarized in Table 2. In $\mathrm{CCl}_{4}$ and xenon only $\tau_{2}$ of the bend vibration can be calculated by this method.

The influence of Coulomb interactions on the relaxation times is relatively weak. Not surprisingly, on average the energy transfer slows down by only about $20 \%$ when the small partial charges are removed (see Table 2). On the other hand, its effect on the branching of the energy flux through symmetric stretch and bend modes of $\mathrm{CO}_{2}$ in $\mathrm{CH}_{2} \mathrm{Cl}_{2}$ is slightly more pronounced. The dashed lines in Fig. 6 indicate that the contribution of the bend energy transfer increases from $74 \%$ to $88 \%$ when the partial charges on the atoms are removed.

\subsection{Equilibrium MD simulations}

According to Landau-Teller theory the CET rate is proportional to the spectral density of the solvent force exerted on the vibrational coordinate of the solute at the frequency of the

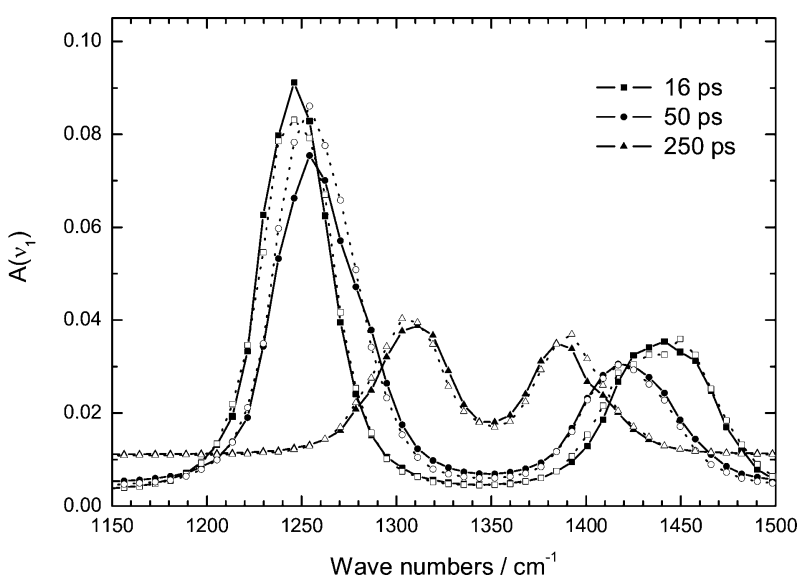

Fig. 5 Temporal evolution of the $\mathrm{CO}_{2}$ symmetric stretch spectra in $\mathrm{CH}_{2} \mathrm{Cl}_{2}$. Filled (opened) symbols linked by solid (dotted) line indicate spectra of the system with (without) particle charges. oscillator. In Figs. 8-10 the corresponding frequency dependent rate constants for $\mathrm{CO}_{2}$ calculated from eqn. (2.8) are shown. In Fig. 8 the solvent dependence of the spectra on the $\mathrm{CO}_{2}$ symmetric stretch (a), the bend (b), and the asymmetric stretch (c) vibration are presented. The plot illustrates that the presence of solvent vibrations dramatically increases CET rate. For xenon the expected slightly stretched exponential decay ${ }^{45}$ of the frequency dependence of the rate constants is observed leading to a practically exclusive contribution of the $\mathrm{CO}_{2}$ lowest frequency vibration (the bend mode) to the energy transfer. In contrast, the spectra of $\mathrm{CCl}_{4}$ and $\mathrm{CH}_{2} \mathrm{Cl}_{2}$ are structured by pronounced resonant features arising from vibrational modes of the solvents. An overlap of $\mathrm{CO}_{2}$ fundamental frequencies with these resonances indicates efficient $\mathrm{V}-\mathrm{V}$ energy transfer. As for xenon the CET rate constant in $\mathrm{CCl}_{4}$ is largest for the bend vibration.

Although in Fig. $8 \mathrm{~b} \nu_{2}$ is close to a strong peak at $790 \mathrm{~cm}^{-1}$ it is not quite clear if $\mathrm{V}-\mathrm{V}$ transfer really dominates this rate constant or if there is also some contribution from $\mathrm{V}-\mathrm{T}$ energy transfer. To answer this question we determined CET rate spectra from EMD simulations of $\mathrm{CO}_{2}$ in rigid $\mathrm{CCl}_{4}$ solvent under similar conditions as for the flexible solvent (see Section 3.3). The result for the $\mathrm{CO}_{2}$ bend vibration is shown in Fig. 9 and compared with $k_{2}(\nu)$ in flexible $\mathrm{CCl}_{4}$ from Fig. 8b. At low frequency both spectra nearly coincide. This part is determined by the mutual translational and rotational motion of $\mathrm{CO}_{2}$ and the solvent which is obviously the same for flexible and rigid $\mathrm{CCl}_{4}$. At $\nu>200 \mathrm{~cm}^{-1}$ the spectra diverge. This is the region where the $\mathrm{CCl}_{4}$ vibrations come into play and determine VER rates. Interestingly, between resonances at 217, 314 and 459 $\mathrm{cm}^{-1}$ etc. the spectrum for flexible $\mathrm{CCl}_{4}$ does not just decay to that for the rigid solvent, indicating that the relaxation rate of the $\mathrm{CO}_{2}$ bend mode for the flexible $\mathrm{CCl}_{4}$ is clearly dominated



Fig. 6 Time dependence of the work performed by $\mathrm{CO}_{2}$ on the solvent $\mathrm{CH}_{2} \mathrm{Cl}_{2}$ with initially unexcited asymmetric stretch mode (solid lines: including electrostatic interactions; dashed lines: neglecting electrostatic interactions) 




Fig. 7 Time dependence of the work performed by $\mathrm{CO}_{2}$ on the solvent $\mathrm{CCl}_{4}$ with initially unexcited asymmetric stretch mode. The notations are the same as in Fig. 6.

by $\mathrm{V}-\mathrm{V}$ transfer. In $\mathrm{CH}_{2} \mathrm{Cl}_{2} \mathrm{~V}-\mathrm{V}$ energy transfer is the prevailing mechanism. Here the spectra are so strongly flattened at high frequency that the symmetric stretch can even compete with $\mathrm{CO}_{2}$ bend energy transfer. This effect can be seen in Fig. 9b where the frequency dependent rate constants in $\mathrm{CH}_{2} \mathrm{Cl}_{2}$ are compared.

The dashed lines in Fig. 8 represent calculations with no partial charges on the atoms. In our case of a non-dipolar solute the influence of Coulomb interactions is not as pronounced as found previously for a polar solute in polar solvents. ${ }^{33,34}$ The strongest effect is seen on the symmetric and asymmetric stretch CET rate constant in $\mathrm{CH}_{2} \mathrm{Cl}_{2}$. Here the Coulomb interaction increases the relaxation rate by a factor of about 2 . The partial charges also strongly increase the resonant structures in the spectra. This is particularly true for bend and asymmetric stretch vibrations of the solute that are

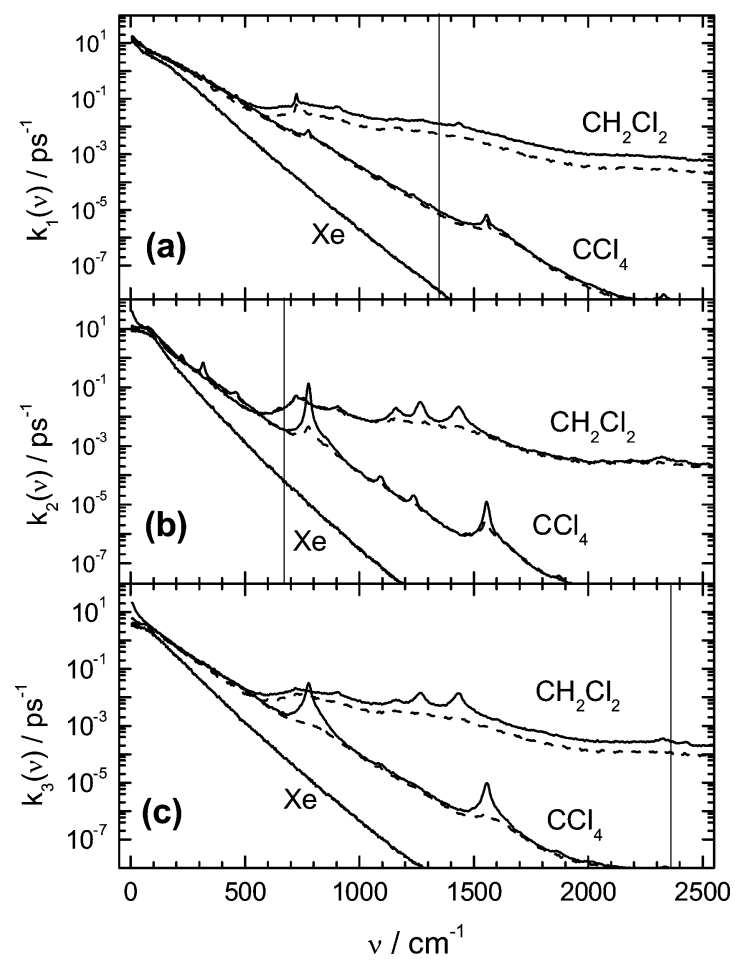

Fig. 8 The frequency dependent VER rate constants (LT approach) of (a) symmetric stretch, (b) bend and (c) asymmetric stretch vibrational modes of $\mathrm{CO}_{2}$ in different solvents. Dashed lines represent the results of simulations with zero partial charges. Additional vertical lines indicate the $\mathrm{CO}_{2}$ fundamental frequencies (see Table 3).

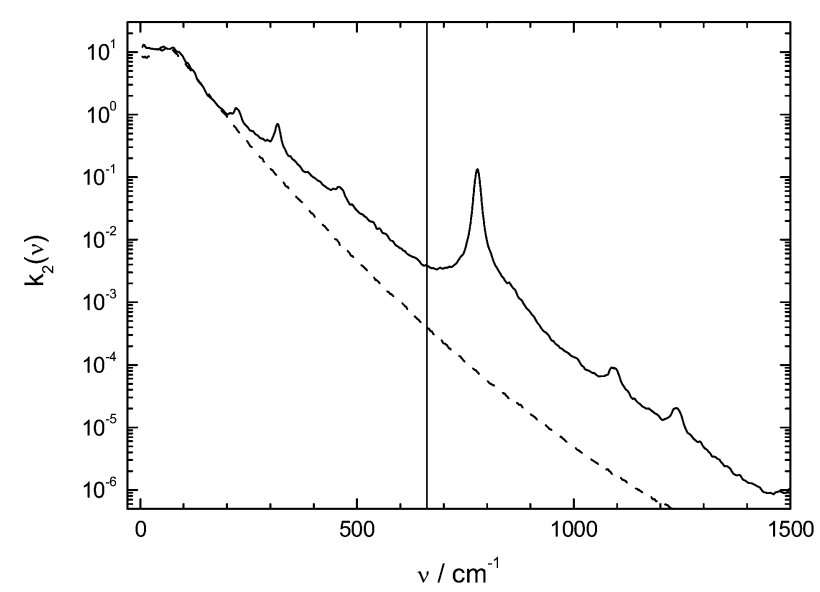

Fig. 9 The frequency dependent VER rate constants of the bend mode $\nu_{2}$ of $\mathrm{CO}_{2}$ in flexible (solid line) and rigid (dashed line) $\mathrm{CCl}_{4}$. The $\mathrm{CO}_{2}$ mode frequency $\nu_{2}=672 \mathrm{~cm}^{-1}$ is shown by a vertical line.

infrared active. Outside the resonance features the spectra are almost the same for charged and uncharged systems.

The mode specific CET time constants of $\nu_{1}$ and $\nu_{2}$ derived from Figs. 8 and 10 are summarized in Table 2. Applying eqn. (2.6) the overall CET time constants were calculated (also shown in Table 2). In general LT and NEMD calculations agree in predicting CET times and energy pathways.

\subsection{Temporal evolution of the antisymmetric stretch spectrum}

To study the evolution of the $\mathrm{CO}_{2}$ asymmetric stretch IR absorption band in $\mathrm{CH}_{2} \mathrm{Cl}_{2}$ a simulated trajectory was divided into pieces of approximately $16 \mathrm{ps}$ duration for each of which the (quasi-stationary) line shape $I(\omega)$ was calculated according to eqn. (2.11) followed by corresponding averaging over all trajectories. In the upper panel of Fig. 11 the results of these calculations are shown. At early times the spectrum is centered at around $2300 \mathrm{~cm}^{-1}$. With decreasing vibrational energy of the $\mathrm{CO}_{2}$ molecule it shifts to the blue by about $80 \mathrm{~cm}^{-1}$ whereas its spectral width decreases from 40 to $20 \mathrm{~cm}^{-1}$. A comparison with Fig. 1a shows that the initial width of the experimental spectrum is much broader. This finding suggests a considerably broader energy distribution of $\mathrm{CO}_{2}$ formed after UV photoinduced fragmentation of organic peroxides than in our simulations. For the simulations this effect can be taken into account by calculating the spectra from 80 ps time intervals (lower panel of Fig. 11). In that case the agreement with the experimental infrared spectra of Fig. 1a is much better. Fig. 2 shows that during the first 80 ps in $\mathrm{CH}_{2} \mathrm{Cl}_{2}$ the $\mathrm{CO}_{2}$ molecules



Fig. 10 The frequency dependent VER rate constants of the vibrational modes of $\mathrm{CO}_{2}$ in flexible $\mathrm{CH}_{2} \mathrm{Cl}_{2}$. The $\mathrm{CO}_{2}$ mode frequencies are shown by vertical lines. 


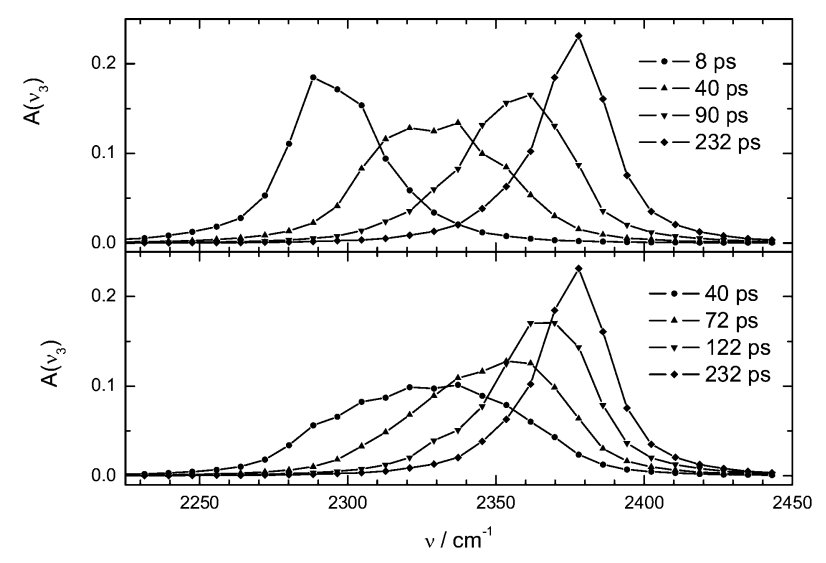

Fig. 11 Temporal evolution of the $\mathrm{CO}_{2}$ infrared absorption spectrum in $\mathrm{CH}_{2} \mathrm{Cl}_{2}$ after averaging over time interval of $16 \mathrm{ps}$ (upper panel) and 80 ps (lower panel), respectively.

lose nearly $60 \mathrm{~kJ} \mathrm{~mol}^{-1}$ of energy which, according to our analysis, would roughly be the width of the internal energy distribution of vibrationally hot $\mathrm{CO}_{2}$ generated in the photofragmentation experiment.

\section{Conclusions}

The results of our simulations and their analysis suggest that ultrafast photofragmentation of organic peroxides generates vibrationally hot carbon dioxide with a broad vibrational energy distribution with most of the energy deposited in the bend and symmetric stretch modes. The modes in $\mathrm{CH}_{2} \mathrm{Cl}_{2}$ and $\mathrm{CCl}_{4}$ relax on a ten to hundred picosecond time scale which is in fair agreement with experiment. The highest frequency asymmetric stretch mode $\nu_{3}$ seems to remain unexcited because in all the solvents considered its vibrational relaxation proceeds on a 10ns time scale, whereas experimentally a picosecond cooling process is observed. The experimentally observed spectral evolution of the $\nu_{3}$-absorption band thus reflects the cooling of the strongly coupled symmetric stretch $\left(\nu_{1}\right)$ and bend $\left(\nu_{2}\right)$ modes due to CET. It is worth noting that according to quantum chemical calculations the $\mathrm{CO}_{2}$ fragment in the acoyloxy radicals is bent and contains increasing amounts of potential energy in the bent coordinate as it progresses along the reaction path towards dissociation. ${ }^{4}$

Comparatively fast vibrational cooling of $\mathrm{CO}_{2}$ in the two polyatomic solvents studied is a consequence of the Fermi resonance between the bend and symmetric stretch vibrations and proceeds by near resonant V-V CET to solvent molecules. This follows from a comparison of CET times obtained from NEMD simulation in $\mathrm{CH}_{2} \mathrm{Cl}_{2}$ (62 ps), $\mathrm{CCl}_{4}$ (344 ps) and $\mathrm{Xe}$ (20 ns), and a consideration of the respective solvent vibrational mode frequencies. The bending vibration of carbon dioxide is much closer to resonance with corresponding vibrational frequencies of dichloromethane (minimum mismatch is $43 \mathrm{~cm}^{-1}$, while three other frequencies are less than $100 \mathrm{~cm}^{-1}$ off, Table 3 ) than with those of carbon tetrachloride (only two vibrational modes are less than $100 \mathrm{~cm}^{-1}$ off resonance). As a consequence, the vibrational cooling rate is a factor of 5 lower in this solvent. The same order of CET rates is observed experimentally.

The LT relaxation rate coefficients extracted from the EMD simulations agree reasonably well with the results of NEMD simulations, showing that the LT-model can be a useful tool for routinely estimating CET-rates in solution. The experimentally observed temporal evolution of the IR-band shape of the $\mathrm{CO}_{2}$ asymmetric stretch mode on the basis of our simulations can be explained by nonlinear interaction with the symmetric stretch and the bend modes. The initially highly vibrationally excited bend mode is characterized by a broad energy distribution which causes a substantial broadening and a substantial red shift of the asymmetric stretch band. In the course of vibrational cooling this band narrows and moves to the position of the asymmetric stretch band of unexcited $\mathrm{CO}_{2}$ molecules.

The extremely small CET rate in Xe obtained from LT calculations is particularly intriguing, as in the case of $\mathrm{V}-\mathrm{T}$ energy transfer the importance of quantum corrections has been generally accepted. For this reason, a comparison of our classical results with experiment appears highly desirable to answer this fundamental issue. Such experiments are underway in our laboratory.

\section{Acknowledgements}

The authors are grateful to Prof. Jürgen Troe for continuously encouraging this work, and to Martin Fechner for providing a constantly friendly computer environment to work in. Thanks go to Martin Sippel and Hans-Martin Loritz for running some of the EMD simulations. Financial support by the Deutsche Forschungsgemeinschaft (Sonderforschungsbereich 357) is gratefully acknowledged.

\section{References}

1 H. Hippler and J. Troe, in Bimolecular Collisions, Advances in GasPhase Photochemistry and Kinetics, eds. M. N. Ashfold and J. Baggot, RSC, London, 1989.

2 G. Flynn, C. Parmenter and A. M. Wodtke, J. Phys. Chem., 1996, 100, 12817

3 B. Abel, J. Assmann, M. Buback, M. Kling, S. Schmatz and J. Schroeder, Angew. Chem. Int. Ed., 2003, 42, 299.

4 B. Abel, J. Assmann, M. Buback, M. Kling, R. Oswald, S. Schmatz, J. Schroeder and T. Witte, J. Phys. Chem. A, 2003, 107, 5157.

5 B. Abel, J. Assmann, M. Buback, C. Grimm, M. Kling, S. Schmatz, J. Schroeder and T. Witte, J. Phys. Chem. A, 2003 , 107, 9499 .

6 B. Abel, J. Assmann, M. Buback, M. Kling, S. Schmatz and J. Schroeder, J. Am. Chem. Soc., 2003, 125, 13274.

7 M. Buback, M. Kling, S. Schmatz and J. Schroeder, Phys. Chem. Chem. Phys., 2004, 6, 5441.

8 J. Aschenbrücker, M. Buback, N. P. Ernsting, J. Schroeder and U. Steegmüller, Ber. Bunsenges. Phys. Chem., 1998, 102, 965.

9 J. Aschenbrücker, M. Buback, N. P. Ernsting, J. Schroeder and U. Steegmüller, J. Phys. Chem. B, 1998, 102, 5552.

10 M. Buback, M. Kling, M. T. Seidel, F.-D. Schott, J. Schroeder and U. Steegmüller, Z. Phys. Chem., 2001, 215, 717.

11 P. Hamm, S. M. Ohline and W. Zinth, J. Chem. Phys., 1997, 106, 519.

12 R. L. Taylor and S. Bitterman, Rev. Mod. Phys., 1969, 41, 26.

13 E. Weitz and G. Flynn, Annu. Rev. Phys. Chem., 1974, 25, 275.

14 C. A. Eckert, B. L. Knutson and P. G. Debenedetti, Nature, 1996, 383, 313 .

15 S. J. V. Frankland and M. Maroncelli, J. Chem. Phys., 1999, 110, 1687.

16 O. Kajimoto, Chem. Rev., 1999, 99, 355.

17 E. F. Smiley and E. H. Winkler, J. Chem. Phys., 1954, 22, 2018.

18 W. Griffith, D. Bricke and V. Blackman, Phys. Rev., 1956, 102, 1209.

19 I. R. Hurle and A. G. Gaydon, Nature, 1959, 184, 1858.

20 D. Wearner, J. F. Roach and W. R. Smith, J. Chem. Phys., 1967, 47, 3096 .

21 C. J. S. M. Simpson, K. B. Bridgman and T. R. D. Chandler, J. Chem. Phys., 1968, 49, 513.

22 C. B. Moore, R. E. Wood, B.-L. Hu and J. T. Yardley, J. Chem. Phys., 1967, 46, 4222.

23 J. T. Yardley and C. B. Moore, J. Chem. Phys., 1967, 46, 4491.

24 E. Fermi, Z. Phys., 1931, 53, 250.

25 D. M. Dennison, Rev. Mod. Phys., 1940, 12, 175.

26 G. Kamimoto and H. Matsuki, J. Chem. Phys., 1970, 53, 3990.

27 T. A. DeTemple, D. R. Suhre and P. D. Coleman, Appl. Phys. Lett., 1973, 22, 349.

28 J. Taine and F. Lepourte, Chem. Phys. Lett., 1979, 65, 554.

29 R. K. Huddleston and E. Weitz, Chem. Phys. Lett., 1981, 83, 174.

30 A. M. Brodnikovsky, S. M. Gladkov and N. I. Koroteev, Opt. Commun., 1982, 40, 312 
31 C. Dang, J. Reid and B. K. Garside, Appl. Phys., 1983, B31, 163.

32 C. Manzanares and G. E. Ewing, J. Chem. Phys., 1978, 69, 1418

33 J. K. Brown, C. B. Harris and J. T. Hynes, J. Chem. Phys., 1988 , 89, 6687.

34 R. M. Whitnell, K. R. Wilson and J. T. Hynes, J. Phys. Chem., 1990, 94, 8625 .

35 R. M. Whitnell, K. R. Wilson and J. T. Hynes, J. Chem. Phys., $1992,96,5354$

36 I. Benjamin and R. M. Whitnell, Chem. Phys. Lett., 1993, 204, 45.

37 C. Heidelbach, V. S. Vikhrenko, D. Schwarzer and J. Schroeder, J. Chem. Phys., 1999, 110, 5286.

38 D. Schwarzer, D. V. Vikhrenko and V. S. Vikhrenko, Chem. Phys., 2004, 300, 197.

39 L. D. Landau and E. A. Teller, Phys. Z. Sowjetunion, 1936, 10, 34

40 R. Zwanzig, J. Chem. Phys., 1961, 34, 1931.

41 D. W. Oxtoby, Adv. Chem. Phys., 1981, 47, 487.

42 S. A. Egorov and J. L. Skinner, J. Chem. Phys., 1996, 105, 7047.

43 R. Rey and J. T. Hynes, J. Chem. Phys., 1996, 104, 2356.

44 (a) C. P. Lawrence and J. L. Skinner, J. Chem. Phys., 2002, 117, 5827; (b) C. P. Lawrence and J. L. Skinner, J. Chem. Phys., 2003, 119, 1623.

45 M. Teubner and D. Schwarzer, J. Chem. Phys., 2003, 119, 2171.

46 S. A. Egorov and J. L. Skinner, Chem. Phys. Lett., 1998, 293, 469.

47 N. J. Wright and N. Makri, J. Chem. Phys., 2003, 119, 1634

48 J. S. Bader and B. J. Berne, J. Chem. Phys., 1994, 100, 8359.

49 D. W. Oxtoby and S. A. Rice, J. Chem. Phys., 1976, 65, 1676.

50 E. J. Heller, E. B. Stechel and M. J. Davis, J. Chem. Phys., 1980, 73, 4720 .

51 C. Heidelbach, J. Schroeder, D. Schwarzer and V. S. Vikhrenko, Chem. Phys. Lett., 1998, 291, 333.
52 C. Heidelbach, V. S. Vikhrenko, D. Schwarzer, I. I. Fedchenia and J. Schroeder, J. Chem. Phys., 1999, 111, 8022.

53 P. H. Berens and K. R. Wilson, J. Chem. Phys., 1981, 74, 4872.

54 H. Ahlborn, B. Space and P. B. Moore, J. Chem. Phys., 2000, 112 , 8083.

55 A. Chedin, J. Mol. Spectrosc., 1979, 76, 430.

56 A. Chedin and J. L. Teffo, J. Mol. Spectrosc., 1984, 107, 333.

57 A. V. Kondratenko, J. Schroeder and V. S. Vikhrenko, Nonlinear Phenomenain Complex Systems, 2002, 6, 502.

58 C. C. Eckart, Phys. Rev., 1935, 47, 552-558.

59 B. Wilson, Jr., J. C. Decius and P. C. Cross, Molecular vibrations: The theory of infrared and Raman vibrational spectra, McGrawHill, New York, 1966.

60 T. Shimanouchi, I. Nakagawa, J. Hiraishi and M. Ishii, J. Mol. Spectrosc., 1966, 19, 78.

61 R. W. Davis, A. G. Robiette and M. C. L. Gerry, J. Mol. Spectrosc., 1981, 85, 399.

62 A. Medina, J. M. M. Roco, A. Calvo Hernandez, S. Welasco, M. O. Bulanin, W. A. Herrebout and B. J. van der Veken, J. Chem. Phys., 2002, 116, 5058.

63 M. Ferrario and M. W. Evans, Chem. Phys., 1982, 72, 141

64 T. Nakagawa, S. Yamanaka, H. Urakawa, K. Kajiwara, H. Maeda and S. Hayashi, J. Mol. Liquids, 1998, 75, 127.

65 NIST: Computational Chemistry Comparison and Benchmark Database, Release 9, 2003 (http://srdata.nist.gov/cccbdb).

66 AutoDock 3.0: http://www.scripps.edu/pub/olson-web/doc/autodock/parameters.html.

67 M. P. Allen and D. J. Tildesley, Computer Simulation of Liquids, Clarendon Press, Oxford, 1987.

68 K. Refson, Moldy User's Manual, (Revision: 2.25.2.6) for release 2.16, 2001, http://www.earth.ox.ac.uk/ keithr/moldy-manual/ moldy.html. 